\title{
ОПТИЧЕСКИЕ СВОЙСТВА МНОГОСЛОЙНЫХ СТРУКТУР НА ОСНОВЕ ТВЕРДЫХ РАСТВОРОВ СИСТЕМЫ CdS-ZnS, ПОЛУЧЕННЫХ МЕТОДОМ ПИРОЛИЗА АЭРОЗОЛЯ
}

\author{
(c) 2017 О. В. Звягина, В. Н. Семенов, Т. В. Самофалова \\ Воронежский государственный университет, Университетская пл., 1, 394018 Воронеж, Россия \\ e-mail: orebenok@mail.ru
}

Поступила в редакцию 01.08.2017 г.

\begin{abstract}
Аннотация. Методом пиролиза аэрозоля растворов тиокарбамидных координационных соединений получены многослойные пленочные структуры на основе твердых растворов системы CdS-ZnS с переменными по толщине концентрациями кадмия и цинка. Исследованы их спектры пропускания, спектры фотолюминесценции и температурная зависимость фототока. Показано, что фототок при длине волны возбуждающего света 380 нм немонотонно зависит от температуры, что связывается с конкуренцией процессов возбуждения и рекомбинации носителей.
\end{abstract}

Ключевые слова: многослойные полупроводниковые структуры, твердые растворы CdS - ZnS, фотолюминесценция, фотопроводимость.

\section{ВВЕДЕНИЕ}

Актуальной задачей современного полупроводникового материаловедения является создание новых простых и недорогих материалов, при синтезе которых легко изменяются физические и химические свойства $[1,2]$. Большое внимание в области фотоэлектроники по-прежнему привлекают халькогениды металлов из-за их способности образовывать твердые растворы и соединения, поскольку свойства этих материалов в первую очередь зависят от их состава.

Цель данной работы - исследование кристаллической структуры и свойств синтезированных методом пиролиза аэрозоля многослойных структур на основе твердых растворов системы CdS-ZnS c последовательным изменением отношений атомных долей ионов кадмия и цинка.

\section{ЭКСПЕРИМЕНТАЛЬНАЯ ЧАСТЬ}

В настоящей работе были получены структуры на основе твердых растворов системы CdS-ZnS, состоящие из 11 слоев разного состава. Для обозначения использовалась запись $\mathrm{ZnS} \rightarrow \mathrm{CdS}$, если слой сульфида кадмия был первым напылен на подложку, в ином случае $(\mathrm{CdS} \rightarrow \mathrm{ZnS})$ многослойная структура формировалась на базе сульфида цинка. Для этого использовался метод пиролиза аэрозоля растворов тиокарбамидных координационных со- единений (ТКС) кадмия и цинка $\left[\mathrm{M}\left(\mathrm{N}_{2} \mathrm{H}_{4} \mathrm{CS}\right)_{2} \mathrm{X}_{2}\right]$ $\left(\mathrm{M}=\mathrm{Cd}, \mathrm{Zn} ; \mathrm{X}=\mathrm{Cl}^{-}, \mathrm{Br}^{-}\right)$[3]. Распыление водных растворов вели на нагретую до $400{ }^{\circ} \mathrm{C}$ ситалловую или кварцевую подложку, причем каждые 15 с напыление прерывалось, и раствор менялся для того, чтобы увеличить или уменьшить содержание ионов $\mathrm{Zn}^{2+}$. Суммарное время напыления образца составляло 165 с. В качестве источников ионов $\mathrm{Cd}^{2+}$ и $\mathrm{Zn}^{2+}$ использовались бромиды и хлориды кадмия и цинка, а донором серы выступала тиомочевина.

Спектр фотолюминесценции измеряли с использованием экспериментальной установки, созданной на базе волоконно-оптического спектрометра USB4000-VIS-NIR (350-1000 нм) фирмы Ocean Optics. B качестве источников возбуждения люминесценции использовали лазерный диод, излучающий на длине волны 405 нм. Плотность мощности излучения не превышала $20 \mathrm{mBT} / \mathrm{cm}^{2}$ для того, чтобы исключить повреждение зерен. Для доставки возбуждающего излучения и сбора сигнала люминесценции использовалось кварцевое оптоволокно диаметром 600 мкм (QP600, Ocean Optics). Измерения проводились в темноте в отсутствие источников рассеянного света. По каждому образцу было зафиксировано около 10 спектров люминесценции, после чего спектры были усреднены.

Исследования фотопроводимости образцов проводились при температуре $23^{\circ} \mathrm{C}$, для возбуждения 
использовался светодиод с длинной волны 380 нм (3.26 эВ), напряжение постоянного тока 5 В.

Рентгенофазовый анализ образцов, осажденных на кварцевых подложках, проводили на рентгеновском дифрактометре PANanalyticalEmpyrean (излучение $\mathrm{CuK \alpha 1)}$. Полученные из дифрактограмм значения межплоскостных расстояний $d$ сравнивались со справочными из базы данных [4].

\section{РЕЗУЛЬТАТЫ И ИХ ОБСУЖДЕНИЕ}

При исследовании многослойной структуры оптическими методами возможно определить ширину запрещенной зоны самого узкозонного слоя $[5,6]$. Говорить об общей ширине запрещенной зоны не корректно, так как полученная структура состоит из отдельных слоев, в каждом из которых добавляются уровни. Каждый слой имеет свое значение $E_{g}$. При индивидуальном исследовании каждого слоя оптическими методами исследования наиболее ярко проявляются твердые растворы на основе CdS, область ZnS имеет большее значение ширины запрещенной зоны и по спектрам поглощения вычисляется сложнее. Легирование образцов приводит к отклонению от литературных данных для монокристаллического CdS и ZnS [7-9]. В данном исследовании наименьшее значение ширины запрещенной зоны характерно для сульфида кадмия. Примеры спектров для многослойных структур, полученных из бромидных ТКС, представлены на рис. 1.
Значения оптической ширины запрещенной зоны для полученных образцов в зависимости от чередования слоев на пути светового луча представлены в табл. 1. Из этих данных видно, что для структуры, в которой слой CdS является последним на пути луча (условное обозначение $\mathrm{ZnS} \rightarrow \mathrm{CdS}$, при регистрации спектра луч падает на слой ZnS), ширина запрещенной зоны увеличена по сравнению с однослойным образцом CdS, полученным из соответствующей соли (2.4 эВ).

Рентгенофазовый анализ показал, что синтезированные многослойные образцы $\mathrm{CdS} \rightarrow \mathrm{ZnS}$ и $\mathrm{ZnS} \rightarrow$ $\mathrm{CdS}$, осажденные из хлоридных и бромидных ТКС, имеют поликристаллическую структуру и кристаллизуются в модификации вюртцита (табл. 2, 3). В ка-

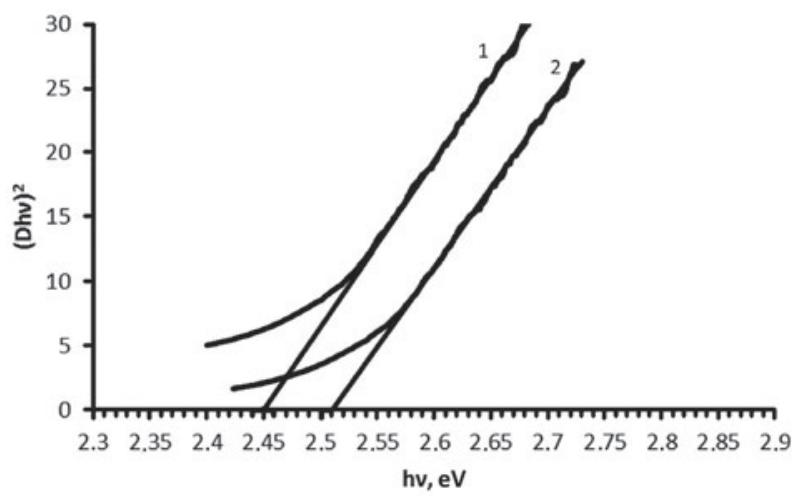

Рис. 1. Спектры поглощения для многослойных структур: 1 - ZnS $\rightarrow \mathrm{CdS} ; 2$ - CdS $\rightarrow$ ZnS

[Fig. 1. Absorption spectra for multilayer structures: 1$\mathrm{ZnS} \rightarrow \mathrm{CdS} ; 2$ - CdS $\rightarrow \mathrm{ZnS}]$

Таблица 1. Оптическая ширина запрещенной зоны $\left(E_{g}\right)$ послойно напыленных образцов системы CdS-ZnS [Table 1. The optical bandgap $\left(E_{g}\right)$ of layer-by-layer dust samples of the CdS-ZnS system CdS-ZnS]

\begin{tabular}{|c|c|c|c|c|}
\hline $\begin{array}{c}\text { Образец } \\
{[\text { Sample }]}\end{array}$ & $\mathrm{ZnS} \rightarrow \mathrm{CdS}(\mathrm{Cl})$ & $\mathrm{ZnS} \rightarrow \mathrm{CdS}(\mathrm{Br})$ & $\mathrm{CdS} \rightarrow \mathrm{ZnS}(\mathrm{Cl})$ & $\mathrm{CdS} \rightarrow \mathrm{ZnS}(\mathrm{Br})$ \\
\hline$E_{g} \cdot$ эB & $2.79 \pm 0.02$ & $2.51 \pm 0.02$ & $2.43 \pm 0.02$ & $2.45 \pm 0.02$ \\
\hline
\end{tabular}

Таблица 2. Межплоскостные расстояния в пленках многослойной структуры $\mathrm{CdS} \rightarrow \mathrm{ZnS}(\mathrm{Cl})$

[Table 2. Interplanar distances in multilayer structures $\mathrm{CdS} \rightarrow \mathrm{ZnS}(\mathrm{Cl})$ ]

\begin{tabular}{|c|c|c|c|c|}
\hline $\begin{array}{c}\text { Номер пика } \\
\text { [Peak Number }]\end{array}$ & $2 \theta$, degree & $d, \AA$ & $\begin{array}{c}\text { Oтнесение } \\
\text { [Structural data] }\end{array}$ \\
\hline 1 & 24.90 & 3.5734 & 7.48 & $\mathrm{Cd}_{0.9} \mathrm{Zn}_{0.1} \mathrm{~S}(100)$ \\
\hline 2 & 26.521 & 3.3581 & 100.00 & $\mathrm{Cd}_{0.8} \mathrm{Zn}_{0.2} \mathrm{~S}(002)$ \\
\hline 3 & 28.288 & 3.1523 & 34.20 & $\mathrm{Cd}_{0.2} \mathrm{Zn}_{0.8} \mathrm{~S}(111)$ \\
\hline 4 & 28.72 & 3.1063 & 24.56 & $\mathrm{Cd}_{0.1} \mathrm{Zn}_{0.9} \mathrm{~S}(111)$ \\
\hline 5 & 36.82 & 2.4391 & 7.16 & $\mathrm{Cd}_{0.3} \mathrm{Zn}_{0.7} \mathrm{~S}(210)$ \\
\hline 6 & 43.88 & 2.0616 & 8.28 & $\mathrm{CdS}_{(220)}$ \\
\hline 7 & 47.94 & 1.8962 & 6.98 & $\mathrm{Cd}_{0.5} \mathrm{Zn}_{0.5} \mathrm{~S}(003)$ \\
\hline 8 & 51.97 & 1.7582 & 9.39 & $\mathrm{Cd}_{0.7} \mathrm{Zn}_{0.3} \mathrm{~S}(200)$ \\
\hline 9 & 52.80 & 1.7325 & 10.25 & $\mathrm{CdS}(201)$ \\
\hline 10 & 54.57 & 1.6802 & 5.60 & $\mathrm{ZnS} \mathrm{(200)}$ \\
\hline
\end{tabular}


Таблица 3. Межплоскостные расстояния в пленках многослойной структуры $\mathrm{ZnS} \rightarrow \mathrm{CdS}(\mathrm{Cl})$

[Table 3. Interplanar distances in multilayer structures $\mathrm{ZnS} \rightarrow \mathrm{CdS}(\mathrm{Cl})]$

\begin{tabular}{|c|c|c|c|r|}
\hline $\begin{array}{c}\text { Номер пика } \\
\text { [Peak Number] }\end{array}$ & 20, degree & $d, \AA$ & $I, \%$ & $\begin{array}{c}\text { Oтнесение } \\
\text { [Structural data] }\end{array}$ \\
\hline 1 & $25.34(4)$ & 3.5119 & 26.88 & $\mathrm{Cd}_{0.8} \mathrm{Zn}_{0.2} \mathrm{~S}(100)$ \\
\hline 2 & $28.51(1)$ & 3.1281 & 100.00 & $\mathrm{Cd}_{0.1} \mathrm{Zn}_{0.9} \mathrm{~S}(111)$ \\
\hline 3 & $44.49(2)$ & 2.0345 & 47.88 & $\mathrm{CdS}(110)$ \\
\hline 4 & $52.49(6)$ & 1.7417 & 35.42 & $\mathrm{ZnS}(103)$ \\
\hline 5 & $54.1(2)$ & 1.6929 & 10.29 & $\mathrm{Cd}_{0.5} \mathrm{Zn}_{0.5} \mathrm{~S}(211)$ \\
\hline
\end{tabular}

честве примера на рис. 2 представлена дифрактограмма образца $\mathrm{CdS} \rightarrow \mathrm{ZnS}$, полученного из хлоридного ТКС.

При сравнении результатов для всех образцов выявлена тенденция, что при формировании образца на CdS, формируется более текстурированная структура, просматривается большее количество

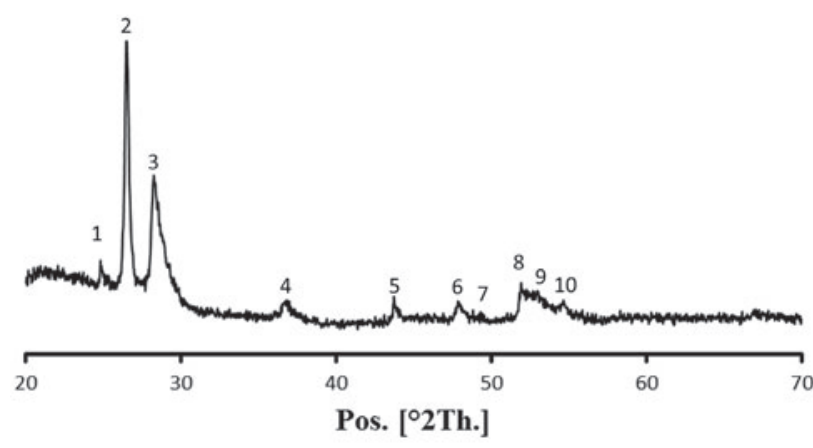

Рис. 2. Дифрактограмма пленки $\mathrm{CdS} \rightarrow \mathrm{ZnS}$

[Fig. 2. Diffractogram of the film CdS $\rightarrow$ ZnS]

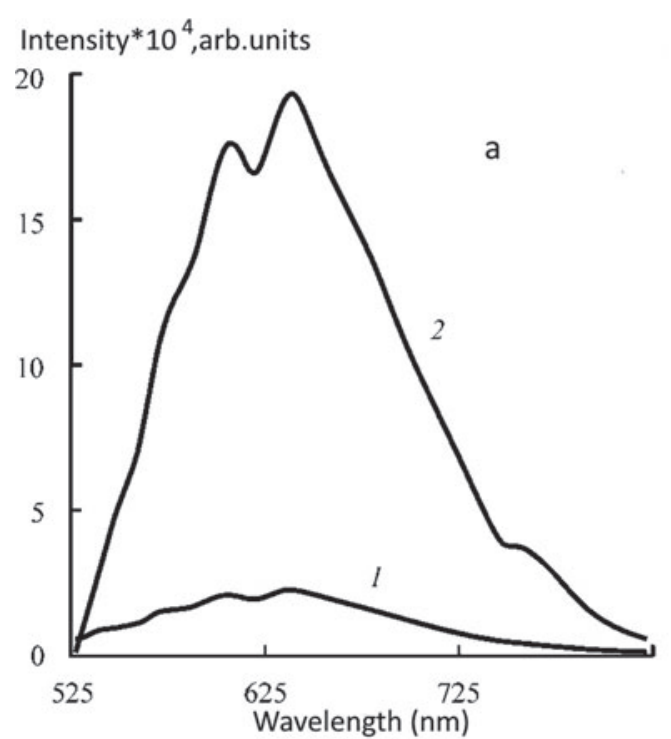

рефлексных пиков и выделяется основное направление роста кристаллов в поликристаллическом образце [002]. При формировании многослойной структуры на основе ZnS выявляется меньшее количество пиков, приоритетное направление кристаллического роста [111].

Сопоставляя результаты рентгенофазового анализа и оптических исследований многослойных структур, можно сделать вывод о том, что при формировании структуры, в которой на подложку первым напыляется слой $\mathrm{CdS}$, ионы $\mathrm{Zn}^{2+}$ диффундируют в нижележащий слой. То же самое справедливо и при диффузии ионов $\mathrm{Cd}^{2+}$ в слой сульфида цинка в образцах $\mathrm{CdS} \rightarrow \mathrm{ZnS}$.

Для того чтобы можно было интерпретировать результаты исследования фотолюминесценции, были изготовлены индивидуальные образцы, которые выступали в качестве эталонных. Спектры фотолюминесценции для многослойных структур представлены рис. 3.

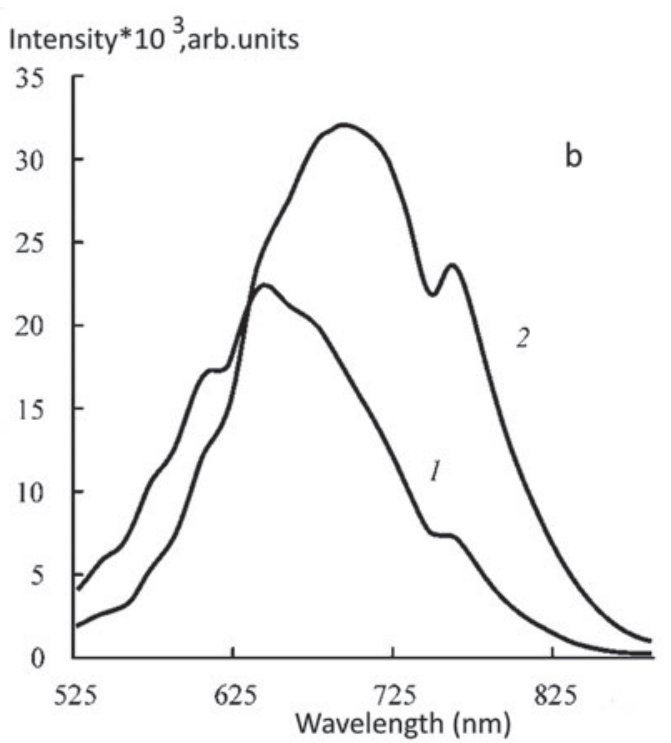

Pис. 3. Спектры фотолюминесценции многослойных структур ZnS $\rightarrow \mathrm{CdS}(a)$ и $\mathrm{CdS} \rightarrow \mathrm{ZnS}(b)$, полученных из бромидных (1) и хлоридных (2) координационных соединений

[Fig. 3. The photoluminescence spectra of multilayer structures $\mathrm{ZnS} \rightarrow \mathrm{CdS}(a)$ And $\mathrm{CdS} \rightarrow \mathrm{ZnS}(b)$, obtained from bromide (1) and chloride (2) Coordination compounds] 
Из рис. 3 видно, что многослойные структуры $\mathrm{CdS} \rightarrow \mathrm{ZnS}$, которые были синтезированы из хлоридных комплексов, имеют максимум интенсивности люминесценции, в несколько раз превышающий интенсивность свечения структур, осажденных из бромидных соединений. Для выделения явных максимумов были построены нормированные спектры, которые представлены на рис. 4 и 5.

Так как исследуемые образцы являются многослойными, полосы люминесценции представляют собой набор простых полос излучения от каждого вида дефекта, которые суммируются и дают представленную на графиках зависимость. В связи с этим интерпретация результатов фотолюминесцентных исследований затруднена.

Из представленных данных видно, что максимумы люминесценции на рис. 4 практически совпадают, что позволяет сделать вывод о том, что в многослойных структурах образовался одинаковый набор дефектов. Проведенные ранее исследования показали, что свечение индивидуальных пленок системы CdS-ZnS обусловлено различными дефектами и комплексами на их основе, например $\left[V_{\mathrm{Cd}} \mathrm{Cl}_{\mathrm{s}}\right]^{\prime},\left[V_{\mathrm{Zn}} \mathrm{Br}_{\mathrm{s}}\right]^{\prime},\left[V_{\mathrm{zn}} \mathrm{O}_{\mathrm{s}}\right]$ и др. [10-13].

Изучение фотоэлектрических свойств показало, что образцы, полученные из бромидных координационных соединений, имеют наибольшую интенсивность фототока $I_{\mathrm{ph}}$.Величины темнового фототока для многослойных структур ZnS $\rightarrow \mathrm{CdS}$ и $\mathrm{CdS} \rightarrow \mathrm{ZnS}$ имеют близкие значения (табл. 4).

Для многослойной структуры, в которой слой $\mathrm{CdS}$ является первым из напыленных на ситалло-

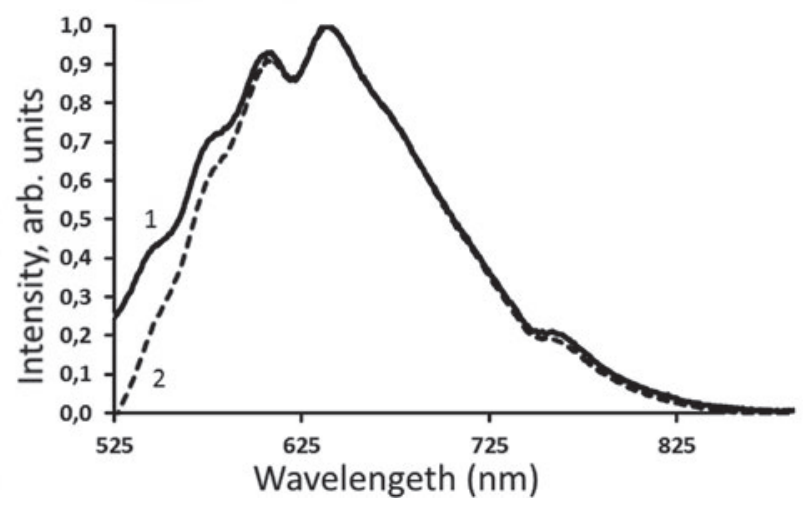

Рис. 4. Графики нормированной зависимости интенсивности люминесценции от длины волны для многослойной структуры $\mathrm{CdS} \rightarrow \mathrm{ZnS}$, полученной из бромидных (1) и хлоридных (2) ТКС

[Fig. 4. Graphs of the normalized dependence of the luminescence intensity on the wavelength for the multilayer structure CdS $\rightarrow$ ZnS obtained from bromide (1) and chloride (2) TCS]
Таблица 4. Фототок для многослойных образцов, полученных из [M( $\left.\left.\mathrm{N}_{2} \mathrm{H}_{4} \mathrm{CS}\right)_{2} \mathrm{Br}_{2}\right]$

[Table 4. Photocurrent for multilayer samples obtained from $\left.\left[\mathrm{M}\left(\mathrm{N}_{2} \mathrm{H}_{4} \mathrm{CS}\right)_{2} \mathrm{Br}_{2}\right]\right]$

\begin{tabular}{|c|c|c|}
\hline $\begin{array}{c}\text { Образец } \\
\text { [Sample] }\end{array}$ & $\begin{array}{c}\text { Темновой } \\
\text { фототок, нA } \\
\text { [Dark } \\
\text { photocurrent, nA] }\end{array}$ & $\begin{array}{c}\text { Стационарный } \\
\text { фототок, нA } \\
\text { [Stationary } \\
\text { photocurrent, nA }\end{array}$ \\
\hline $\mathrm{CdS} \rightarrow \mathrm{ZnS}(\mathrm{Br})$ & $0.20 \pm 0.02$ & $1.42 \pm 0.02 \cdot 10^{3}$ \\
\hline $\mathrm{ZnS} \rightarrow \mathrm{CdS}(\mathrm{Br})$ & $0.18 \pm 0.02$ & $9.14 \pm 0.02 \cdot 10^{3}$ \\
\hline $\mathrm{CdS}$ & $0.48 \pm 0.02$ & $3.16 \pm 0.02 \cdot 10^{3}$ \\
\hline
\end{tabular}

вую подложку, a ZnS последним, была измерена температурная зависимость фототока.

Исследования проводились при той же длине волны возбуждающего света, и температурах, начиная от комнатной. Выход на стационарные значения фототока происходил в течение 30 мин, затем образец нагревался до следующей температуры. Зависимость фототока от температуры представлена на рис. 6.

Немонотонную температурную зависимость фототока можно объяснить следующим образом. При комнатной температуре основной вклад в фотопроводимость полупроводника вносят фотовозбужденные электроны, которые переходят из валентной зоны в зону проводимости. Можно предположить, что при незначительном увеличении температуры вклад в фототок будут вносить также электроны, возбужденные с донорных уровней. При дальнейшем повышении температуры начинают конкурировать процессы рекомбинации, вследствие чего наблюдается падение

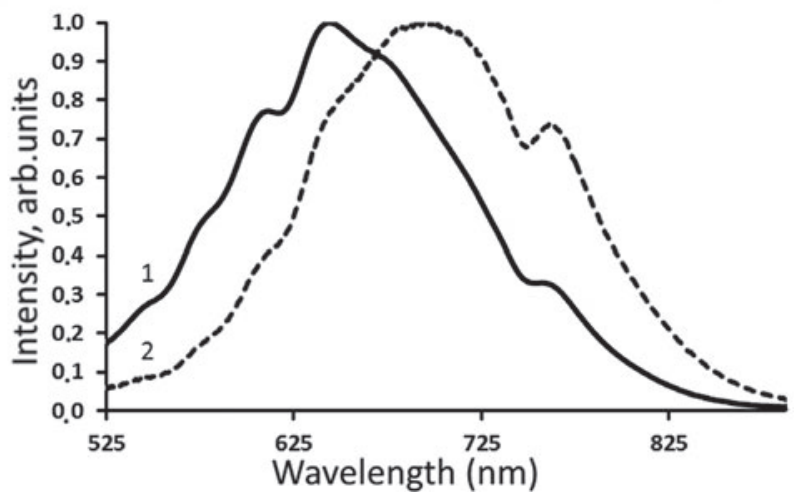

Рис. 5. График нормированной зависимости интенсивности люминесценции от длинны волны для многослойной структуры $\mathrm{ZnS} \rightarrow \mathrm{CdS}$, полученной из бромидных (1) и хлоридных (2) ТКС

[Fig. 5. Graphs of the normalized dependence of the luminescence intensity on the wavelength for the multilayer structure $\mathrm{ZnS} \rightarrow \mathrm{CdS}$ obtained from bromide (1) and chloride (2) TCS] 


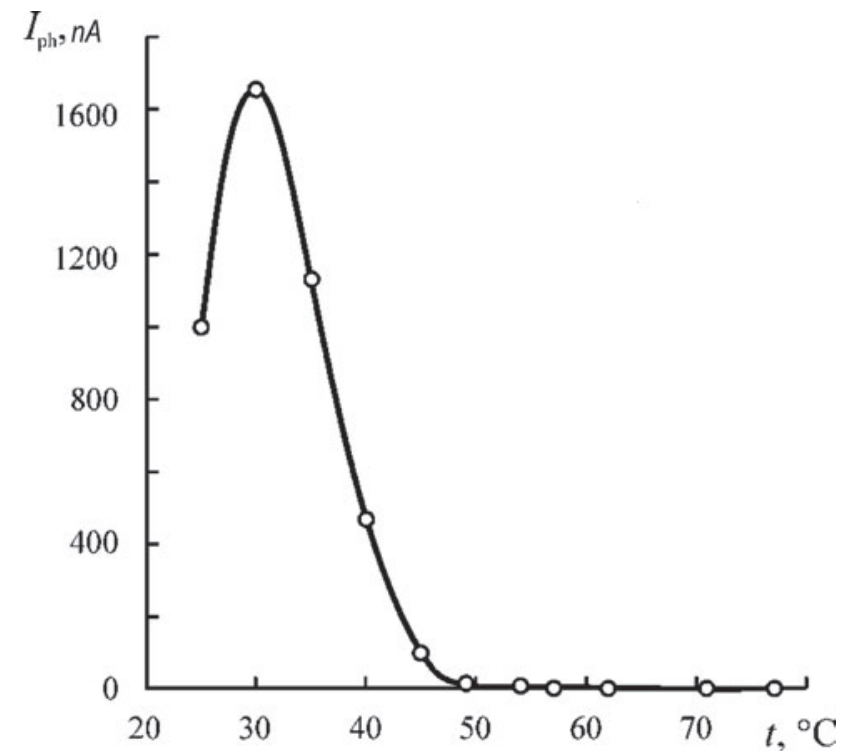

Рис. 6. Зависимость фототока от температуры для многослойной структуры $\mathrm{ZnS} \rightarrow \mathrm{CdS}$, полученной из бромидных ТКС

[Fig. 6. Dependence of the photocurrent on the temperature for a multilayer structure $\mathrm{ZnS} \rightarrow \mathrm{CdS}$, obtained from bromide TCS]

фототока до темнового значения. В результате на температурной зависимости фотопроводимости появляется максимум стационарного фототока.

Такое поведение фотопроводимости предполагает возможность создания полупроводниковых датчиков света или других видов электромагнитного излучения. Синтезированные структуры могут быть использованы для измерения освещенности и в качестве компонентов для изготовления преобразователей солнечной энергии.

\section{ЗАКЛЮЧЕНИЕ}

Пиролизом аэрозоля растворов тиомочевинных координационных соединений $\left[\mathrm{M}\left(\mathrm{N}_{2} \mathrm{H}_{4} \mathrm{CS}\right)_{2} \mathrm{X}_{2}\right]$ синтезированы многослойные структуры заданного состава CdS $\rightarrow \mathrm{ZnS}$ (а) и $\mathrm{ZnS} \rightarrow \mathrm{CdS}$ (б):

a) $\mathrm{CdS}-\mathrm{Cd}_{0,9} \mathrm{Zn}_{0,1} \mathrm{~S}-\mathrm{Cd}_{0,8} \mathrm{Zn}_{0,2} \mathrm{~S}-\mathrm{Cd}_{0,7} \mathrm{Zn}_{0,3} \mathrm{~S}-$ $\mathrm{Cd}_{0,6} \mathrm{Zn}_{0,4} \mathrm{~S}-\mathrm{Cd}_{0,5} \mathrm{Zn}_{0,5} \mathrm{~S}-\mathrm{Cd}_{0,4} \mathrm{Zn}_{0,6} \mathrm{~S}-\mathrm{Cd}_{0,3} \mathrm{Zn}_{0,7} \mathrm{~S}-$ $\mathrm{Cd}_{0,2} \mathrm{Zn}_{0,8} \mathrm{~S}-\mathrm{Cd}_{0,1} \mathrm{Zn}_{0,9} \mathrm{~S}-\mathrm{ZnS}$;

б) ZnS-Cd ${ }_{0,1} \mathrm{Zn}_{0,9} \mathrm{~S}-\mathrm{Cd}_{0,2} \mathrm{Zn}_{0,8} \mathrm{~S}-\mathrm{Cd}_{0,3} \mathrm{Zn}_{0,7} \mathrm{~S}-$ $\mathrm{Cd}_{0,4} \mathrm{Zn}_{0,6} \mathrm{~S}-\mathrm{Cd}_{0,5} \mathrm{Zn}_{0,5} \mathrm{~S}-\mathrm{Cd}_{0,6} \mathrm{Zn}_{0,4} \mathrm{~S}-\mathrm{Cd}_{0,7} \mathrm{Zn}_{0,3} \mathrm{~S}-$ $\mathrm{Cd}_{0,8} \mathrm{Zn}_{0,2} \mathrm{~S}-\mathrm{Cd}_{0,9} \mathrm{Zn}_{0,1} \mathrm{~S}-\mathrm{CdS}$.

Установлено, что наибольшая интенсивность фотолюминесценции наблюдается для слоев, осажденных из ТКС $\left[\mathrm{M}\left(\mathrm{N}_{2} \mathrm{H}_{4} \mathrm{CS}\right)_{2} \mathrm{CI}_{2}\right]$. Для всех образцов зафиксировано уширение полос люминесценции.

Результаты рентгенофазового анализа показали, что все осажденные в многослойной структу- ре слои имеют модификацию вюрцита, являются поликристаллическими и имеют выраженное направление кристаллизации.

Многослойные структуры, синтезируемые из $\left[\mathrm{M}\left(\mathrm{N}_{2} \mathrm{H}_{4} \mathrm{CS}\right)_{2} \mathrm{Br}_{2}\right]$, имеют значение стационарного тока сопоставимое со значениями для индивидуального CdS, синтезируемого в аналогичных условиях.

Оптические исследования показали, что спектры пропускания не отражают край собственного поглощения для каждого осажденного слоя.

Значение ширины запрещенной зоны для образцов CdS $\rightarrow \mathrm{ZnS}$ несколько увеличена при сравнении с индивидуальным CdS. Многослойные образцы ZnS $\rightarrow \mathrm{CdS}$, синтезируемые из хлоридных координационных соединений, обладают большей оптической шириной по сравнению с образцами из бромидных ТКС.

Результаты исследований получены на оборудовании Центра коллективного пользования научным оборудованием ВГУ.

\section{СПИСОК ЛИТЕРАТУРЫ}

1. Thahab S. M. // Materials Science in Semiconductor Processing, 2014, № 26, p. 49-54.

2. Бурмаева Н. В. Основы полупроводниковой электроники. М.:, Физматлит, 2012, 310 с.

3. Семенов В. Н., Наумов А. В. // Вестн. ВГУ. Серия химия, биология, 2000, № 2, с. 50-55.

4. Powder Diffraction File. Swarthmore: Joint Committee on Powder Diffraction Standards, 1996.

5. Уханов Ю. М. Оптические свойства полупроводников. М.: Наука, 1977, 366 с.

6. Кнотько А. В. Химия твердого тела: учеб. пособие для студ. высш. учеб. заведений, М.: Издательский центр «Академия», 2006, 304 с.

7. Наумов А. В, Болгова Т. Г., Семенов В. Н. и др. // Heopz. матер. 2000, т. 42, № 5, с. 523-529.

8. Самофалова Т. В., Семенов В. Н. // Вестн. ВГУ. Серия химия, биология, 2015, № 1, с. 42-48.

9. Нитута А. Н., Ребенок О. В., Такмакова Е. В. // Тезисы докладов 7-ой всероссийской конференции «Физико-химические процессы в конденсированных средах и на межфазных границах», 2015, с. 245-246.

10. Самофалова Т. В., Семенов В. Н. // Конденсированные среды и межфазные гранищы, 2016, т. 18, № 2, c. 248-255.

11. Самофалова Т. В., Семенов В. Н. // Конденсированные среды и межфазные границы, 2007, т. 9, № 2, c. 152-155.

12. Метелева Ю. В // Неорган. Мат., 2001, т. 37, № 12, c. $1435-1438$.

13. Лукин А. Н., Самофалова Т. В., Семенов В. Н., Наумов А. В. // Известия ВУЗов: Физика, 2011, т. 54, № 2/2, c. 211-215. 


\title{
OPTICAL PROPERTIES OF MULTILAYER STRUCTURES ON THE BASIS OF SOLID SOLUTIONS OF THE SYSTEM CdS-ZnS, OBTAINED BY THE METHOD OF PYROLYSIS OF AEROSOL
}

\author{
(C) 2017 O. V. Zvyagina, V. N. Semenov, T. V. Samofalova \\ Voronezh State University, 1 Universitetskaya sqr., 394018 Voronezh, Russia \\ e-mail: orebenok@mail.ru
}

Received 01.08.2017

\begin{abstract}
Multilayer film structures based on solid solutions of the CdS-ZnS system with variable thickness concentrations of cadmium and zinc were obtained by the pyrolysis of aerosol solutions of thiocarbamide coordination compounds. Their transmission spectra, the photoluminescence spectra, and the temperature dependence of the photocurrent are studied. It is shown that the photocurrent at the wavelength of the exciting light $(380 \mathrm{~nm}$ ) is not monotonically dependent on the temperature, which is associated with the competition of carrier excitation and recombination processes

The purpose of this work is to study the crystal structure and properties of multilayer structures synthesized by pyrolysis of aerosol on the basis of solid solutions of the CdS-ZnS system with successive changes in the ratios of the atomic fractions of cadmium and zinc ions

It is established that the highest photoluminescence intensity is observed for layers precipitated from TCS $\left[\mathrm{M}\left(\mathrm{N}_{2} \mathrm{H}_{4} \mathrm{CS}\right)_{2} \mathrm{CI}_{2}\right]$. For all the samples, the luminescence bands broadened.

The results of $\mathrm{X}$-ray phase analysis showed that all the layers deposited in the multilayer structure have a wurtzite modification, are polycrystalline, and have a pronounced crystallization direction. The multilayer structures synthesized from $\left[\mathrm{M}\left(\mathrm{N}_{2} \mathrm{H}_{4} \mathrm{CS}\right)_{2} \mathrm{Br}_{2}\right]$ have a steady-state current value comparable to the values for individual CdS synthesized under similar conditions.

Optical studies have shown that the transmission spectra do not reflect the intrinsic absorption edge for each deposited layer.

The value of the bandgap width for CdS $\rightarrow$ ZnS samples is slightly increased when compared with individual CdS. Multilayer $\mathrm{ZnS} \rightarrow \mathrm{CdS}$ samples synthesized from chloride coordination compounds have a greater optical width compared to samples from bromide TCS.
\end{abstract}

Keywords: multilayer semiconductor structures, solid solutions of CdS-ZnS, photoluminescence, photoconductivity.

\section{ACKNOWLEDGMENTS}

The research results were obtained with equipment of Voronezh State University Centre for Collective Use of Scientific Equipment

\section{REFERENCES}

1. Thahab S. M. Materials Science in Semiconductor Processing, 2014, no. 26, pp. 49-54. doi.org/10.1016/j. mssp.2014.04.005

2. Burmaeva N. V. Fundamentals of Semiconductor Electronics. Moscow, Fizmatlit Publ., 2012, 310 p. (in Russian)

3. Semenov V. N., Naumov A. V. Proceedings of Voronezh State University. Series: Chemistry. Biology. Pharmacy, 2000, no. 2, pp. 50-55. Available at: http://www. vestnik.vsu.ru/pdf/chembio/2000/02/semenov.pdf

4. Powder Diffraction File. Swarthmore: Joint Committee on Powder Diffraction Standards, 1996.

5. Uhanov Yu. M. Optical Properties of Semiconductors. Moscow, Science Publ. 1977, 366 p. (in Russian)
6. Knotko A. V. Solid State Chemistry: Textbook. Allowance for stud. Supreme. Training. Institutions, M.: Publishing Center “Academy” Publ., 2006, 304 p. (in Russian)

7. Naumov A. V., Bolgova T. G., Semenov V. N., et al. Inorg. Mater., 2000, vol. 42, no. 5, pp. 523-529.

8. Samofalova T. V., Semenov V. N., Takmakova E. V., Ovechkina N. M. Proceedings of Voronezh State University. Series: Chemistry. Biology. Pharmacy, 2015, no. 1, pp. 42-48. Available at: http://www.vestnik.vsu.ru/pdf/ chembio/2015/01/2015-01-07.pdf (in Russian)

9. Nituta A. N., Rebenok O. V., Takmakova E. V. "Physicochemical Processes in Condensed Matter and on Interphase Boundaries", Proceedings of the 7-th All-Russian Conference, 2015, p. 245-246.

10. Samofalova T. V., Semenov V. N. Condensed Matter and Interphase, 2016, vol. 18, no. 2, pp. 248-255. Available at: http://www.kcmf.vsu.ru/resources/t_18_2_2016_ 009.pdf (in Russian)

11. Samofalova T. V., Meteleva Yu. V., Naumov A. V., Semenov V. N., Novikov G. F. Condensed Matter and Inter- 
phase, 2007, vol. 9, No. 2, pp. 152-155. Available at: http://www. kcmf.vsu.ru/resources/t_09_2_2007_011.pdf (in Russian)

12. Meteleva Yu. V. Inorg. Mater., 2001, vol. 37, no. 12, pp. 1435-1438.
13. Lukin A. N., Samofalova T. V., Semenov V. N., Naumov A. V. News of Higher Educational Institutions: Physics, 2011, vol. 54, no. 2/2, pp. 211-215.
Звягина Ольга Владимировна - магистрант химического факультета, Воронежский государственный университет; тел.: +7(951) 8781317, e-mail: orebenok@ mail.ru

Семенов Виктор Николаевич - д. х. н., заведующий кафедрой общей и неорганической химии, Воронежский государственный университет; тел.: +7(473) 2208610, e-mail: office@chem.vsu.ru

Самофалова Татьяна Владимировна - к. х. н., ассистент кафедры общей и неорганической химии, Воронежский государственный университет; тел.: +7(473) 2208610, e-mail: TSamofalova@bk.ru
Zvyagina Olga $V$. - the competitor for science degree of Master Science in Chemistry, Voronezh State University; ph.: +7(951) 8781317, e-mail: orebenok@mail.ru

Semenov Victor N. - Dr. Sci. (Chem.), Head of General and Inorganic Chemistry, Voronezh State University; ph.: +7(473) 2208610, e-mail: office@chem.vsu.ru

Samofalova Tatyana V. - Cand. Sci. (Chem.), Assistant Lecturer, Department of General and Inorganic Chemistry, Voronezh State University; ph.: +7(473) 2208610, e-mail: TSamofalova@bk.ru 